\title{
Cardiopulmonary Bypass Alone Does Not Cause Postoperative Cognitive Dysfunction Following Open Heart Surgery
}

\author{
Ratna Farida Soenarto ${ }^{1,{ }^{*}}$, Arif Mansjoer ${ }^{2}$, Nurmiati Amir ${ }^{3}$, Maipe Aprianti ${ }^{1}$ and Aries Perdana ${ }^{1}$ \\ ${ }^{1}$ Department of Anesthesiology and Intensive Care, Faculty of Medicine, University of Indonesia, Jakarta, Indonesia \\ ${ }^{2}$ Department of Internal Medicine, Faculty of Medicine, University of Indonesia, Jakarta, Indonesia \\ ${ }^{3}$ Department of Psychiatry, Faculty of Medicine, University of Indonesia, Jakarta, Indonesia \\ "Corresponding author: Department of Anesthesiology and Intensive Care, Faculty of Medicine, University of Indonesia, Jakarta, Indonesia. Email: fida.soenarto@gmail.com
}

Received 2018 August 25; Revised 2018 October 24; Accepted 2018 October 30.

\begin{abstract}
Background: Postoperative cognitive dysfunction (POCD) is commonly observed following cardiac surgery. The utilization of cardiopulmonary bypass ( $\mathrm{CPB}$ ) is associated with many possible mechanisms to cause POCD. However, there is no evidence confirming that $\mathrm{CPB}$ alone is the cause of POCD.

Objectives: The current study aimed at evaluating several factors suspected to cause POCD following cardiac surgery in Cipto Mangunkusumo Hospital, Jakarta, Indonesia.

Methods: The current prospective cohort study was conducted on 54 patients who were candidates for cardiac surgery. The assessment of memory, attention, and executive functions was performed by neuropsychological tests, before and after the surgery. Cognitive decline was defined as a $20 \%$ decrease in cognitive function in at least one of the tests. Inclusion criteria were adults spokeing Bahasa Indonesia fluently, literate, and giving consent to participate in the study. The analyzed risk factors included age, diabetes, educational level, duration of aortic cross clamp, and duration of cardio-pulmonary bypass.

Results: POCD occured in $40.7 \%$ of subjects that underwent cardiac surgery using cardiopulmonary bypass. Age was the only influential factor through bivariate test and logistic regression analysis $(\mathrm{P}=0.001)$. The current study conducted a logistic regression test on age variable; the obtained result indicated an increasing trend of POCD in accordance with age group.

Conclusions: Durations of CPB, cross clamp, diabetes, and educational level were not the main risks of POCD. Old age was a significant predictor to POCD.
\end{abstract}

Keywords: POCD, Cognitive Function, Cardiopulmonary Bypass, Open Heart Surgery

\section{Background}

Cognitive function includes receiving, analyzing, storing, and reclaiming the process of sensory input (1). In general, cognitive function comprises attention, language, memory, motoric, and executive functions (2). The mechanism of perception allows individuals to perceive knowledge and solve problems in daily life. Any cognitive dysfunction is normally followed by confusion, hallucination, and delirium (3).

Cardiopulmonary bypass is normally conducted during open heart surgery allowing the surgeon to evaluate the heart chambers $(4,5)$. The machine and the patient are connected through synthetic lines and tubes. The blood from the heart is delivered to the machine to undergo oxygen and carbondioxide diffusion $(5,6)$. However, this machine may cause many undesirable side effects due to the alteration in normal circulation and perfusion. There are many complications regarding the cardiopulmonary bypass machine, including the central nervous system (CNS) problems.

There are various complications in CNS, including postoperative cognitive dysfunction (7). Many patients that undergo open heart surgery are in productive age, in which cognitive function is essential for daily activity. The mechanism of cognitive dysfunction is poorly known. There are three possibilities including inadequate brain perfusion, systemic inflammatory reactions caused by cardiopulmonary bypass, and microemboli, which impairs brain perfusion $(8,9)$. Previous studies mentioned the risk factor of POCD including patient factors (age, education, comorbid), surgical factors (hypothermia, cardiopulmonary bypass, cross-clamp duration, and bleeding), anesthesia factors (hypotension, anesthetics), and postoperative factors (rehabilitation, depression, social support) (8). 
Age is mentioned as one of major predictors of POCD. Previsou studies mentioned that increased age was related to higher incidence of POCD, especially within six months postoperatively (10). The relationship between duration of cardiopulmonary bypass and POCD is still unknown due to the controversy of the results. However, longer duration of cardiopulmonary bypass would result in more microemboli (11). Cross-clamp duration might cause hypoperfusion and release microemboli leading to impaired cerebral blood flow.

The impact of POCD might occur a long time after the patient is discharged. Early assessment of the cognitive function as well as early detection of POCD is required. Therefore, interventions could be conducted to prevent any cognitive impairments postoperatively $(2,12)$.

In Cipto Mangunkusumo Hospital, cardiac surgery is conducted routinely since 2003. From 2007 to 2017, about 4326 patients underwent cardiac surgery and in 3400 of the cases the cardiopulmonary bypass machine was employed. There was no specific record on the incidence of POCD after surgery; however, there were some incidences of stroke postoperatively. This complication may impact the quality of life of the patients later on. Early detection of any predictors of POCD should be conducted to prevent such events. However, inadequate studies identified the predictors of POCD in patients undergoing cardiac surgery.

\section{Objectives}

The current study aimed at identifying the risk factors of impaired cognitive function, including age, education level, diabetes mellitus, duration of cardiopulmonary bypass, and cross-clamp, in patients that underwent cardiac surgery in Cipto Mangunkusumo Hospital, Jakarta, Indonesia.

\section{Methods}

The current prospective cohort study was conducted from November 2016 to March 2017 in Cipto Mangunkusumo Hospital. The subjects were adult candidates for cardiac surgery with cardiopulmonary bypass, literate, able to speak Bahasa fluently that agreed to participate in the study. The exclusion criteria were the history of cognitive and psychiatric illnesses such as delirium, dementia, amnestic or depression, current consumption of psychotropic drugs, and impaired dominant hands. All subjects that met the inclusion and exclusion criteria were enrolled in the study.
The independent variables were age, education level, diabetes mellitus, cardiopulmonary bypass, and crossclamp duration; while the dependent variable was decreased cognitive function. The cognitive functions assessed in the current study were memory, attention, and executive function. For memory function, the employed instrument was immediate and delayed Rey AuditoryVerbal Learning Test (13). For attention function, the employed instrument was digit span forward and backward (14). Lastly, for executive function, the employed instrument was trial making test $A$ and $B(15)$.

The study subjects underwent cognitive function assessment one day before surgery. Postoperative cognitive function assessment was measured on the fifth day postoperative or on the last day of hospital stay. Postoperative cognitive dysfunction was defined as $20 \%$ decrease in cognitive function test in at least one of the cognitive function tests assessed in the current study. The data were analysed by statistical calculation with SPSS version 21.0 released by IBM in the United States.

\section{Results}

Out of the 70 candidates for cardiac surgery in Cipto Mangunkusumo Hospital in the study period, 54 subjects met the inclusion criteria. The majority of the subjects were younger than 65 years old ( $81 \%$ vs. 19\%) and $70.4 \%$ of the subjects were male. Mean body mass index (BMI) was $24 \pm 4.3 \mathrm{~kg} / \mathrm{m}^{2}$. Most of the subjects had high school diploma ( $81 \%$ vs $19 \%$ ). Most subjects were candidates for coronary artery bypass graft (CABG) surgery (69\%). The number of subjects with duration of cardiopulmonary bypass $<120$ and $>120$ minutes were $59 \%$ vs. $41 \%$, respectively. Similarly, the number of subjects with duration of cardiopulmonary bypass $<90$ minutes and $>90$ minutes were $56 \%$ vs. $44 \%$, respectively. According to the cognitive function test results, cognitive function decreased in $40.7 \%$ of the subjects, following the cardiac surgery.

Based on Table 1, it was observed that age was significantly associated with cognitive function. Patients aged more than 65 years were associated with decreased cognitive function. However, other variables such as education level, diabetes mellitus, duration of cardiopulmonary bypass, and duration of cross-clamp were not associated with cognitive function.

The current study observed decreased cognitive function before surgery in $37 \%$ of the subjects, which mostly had declined memory function followed by executive and attention function (54.8\%, 29\%, and $16.1 \%$, respectively). However, postoperatively, the most declined parameter 


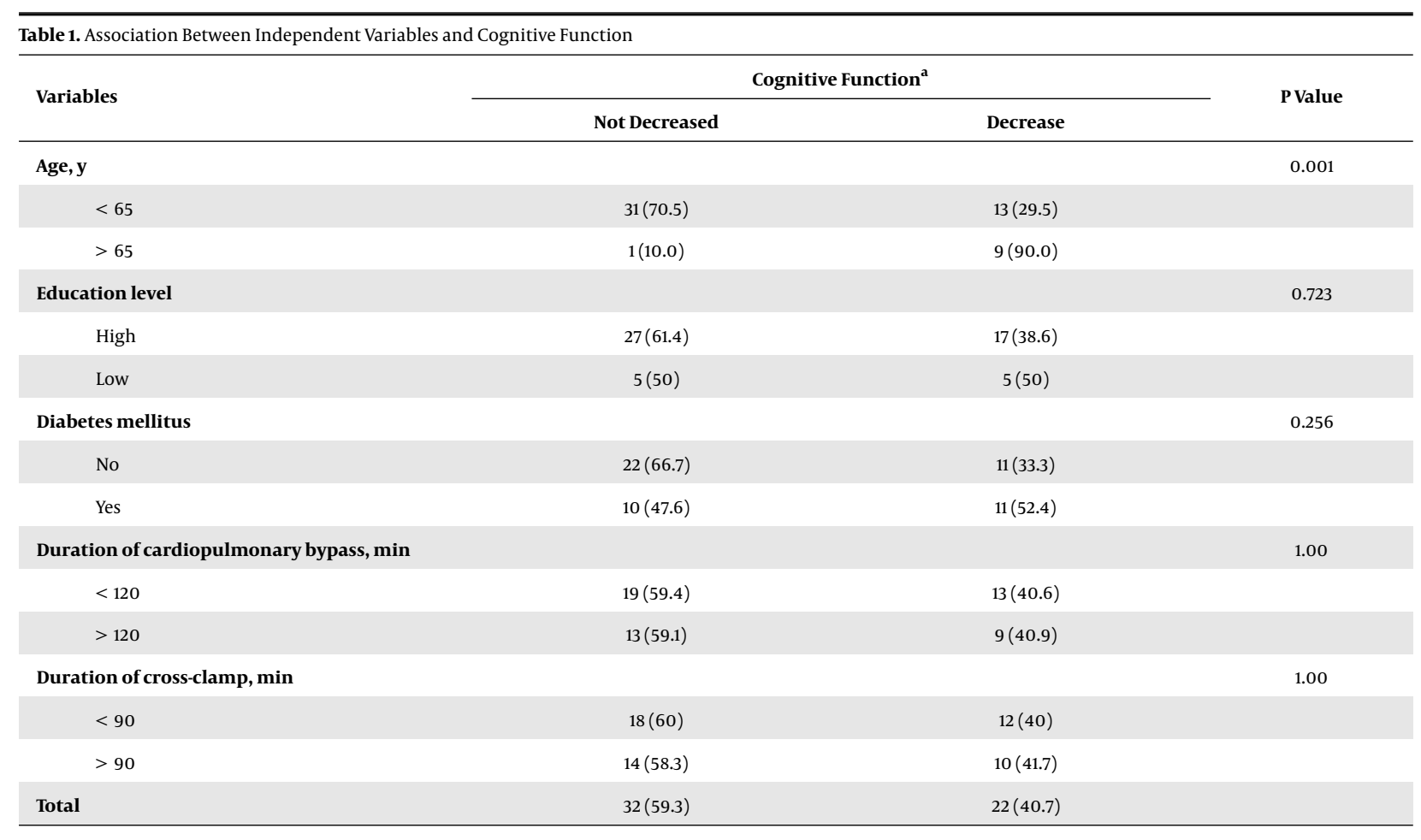

${ }^{\mathrm{a}}$ Values are expressed as No. (\%).

was attention function (38.5\%). No mortality or major neurological dysfunction was reported during the current study.

\section{Discussion}

The current prospective study was conducted on 54 subjects who were candidates for cardiac surgery with cardiopulmonary bypass. Results of the study showed that the incidence of POCD on the last day of hospital stay was 40.7\%. Previous studies also reported similar results, which was $39.8 \%$ and $44.5 \%$ of all patients that underwent cardiac surgery $(16,17)$. POCD remains as one of the most common complications postoperatively. The incidence of POCD ranged 30\% to $79 \%$ in the literature. Newman et al. mentioned that the incidence of POCD following cardiac surgery reached its peak on the last day of hospital stay, which was $53 \%$ (12).

Aging is marked by degenerative changes with decreased functional capacity (18). This process happens in all the system organs regardless of other comorbids. Results of the current study showed that geriatric patients had higher risk to develop POCD $(\mathrm{P}<0.05)$. This finding was similar with those of other studies that mentioned age as a significant risk factor for POCD among patients under- going CABG surgery (12). Moller et al. mentioned that patients aged more than 60 years had high risk to develop short-term POCD, which was a strong predictor for longterm POCD (19).

The current study developed linear regression analysis for age variable as shown in Figure 1. Results of this analysis showed that the risk of POCD increased as the age increased. The impact of aging process in the central nervous system includes decreased brain mass, neuron density, and neuron complexity. The number of serotonin, acethylcholine, and dopamine receptors also decrease along with increased postsynaptic degradation. These changes limit the capacity of the brain to respond to any stress including anesthesia and surgical stress (20). Hemodynamic changes during surgery might cause impaired cerebral blood flow leading to cerebral ischemia18. This might cause neuronal cell death and induce ischemic cascade. The cascade results in glutamate release, catabolic enzyme activation, free radicals production, apoptosis reaction, and inflammation (21). In the current study, $60 \%$ of geriatric patients already had low cognitive score and the majority of geriatric patients had significant decrease of cognitive function.

The current study also found that the number of POCD was higher among subjects with low education, which was 


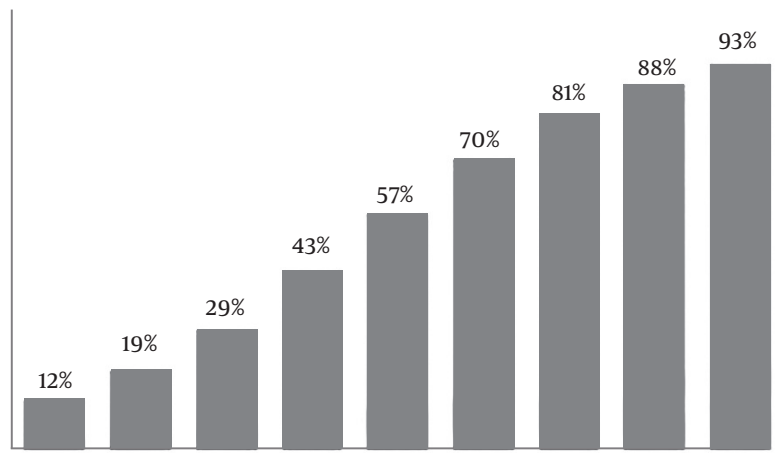

Age 40 Age 45 Age 50 Age 55 Age 60 Age 65 Age 70 Age 75 Age 80

Figure 1. Linear regression of cognitive decline per age group

not statistically significant. Ziyaeifard et al. also reported a similar finding (17). This might be due to limited classification of education level in the current study. Meanwhile, other studies stated that low education was more vulnerable to nervous system damage in relation with nutrition, alcoholism, comorbidity, and trauma (12). Subjects with higher education might be able to conduct better compensation strategy in the behavioural and neuronal levels (22).

Diabetes mellitus is a complex metabolic disease, which contributes to many diseases including end-stage renal disease, neuropathy, and cardiovascular diseases (23). The brain utilizes $25 \%$ of the total glucose in the body (24). Therefore, during hypoglycemic period, cell metabolism of the brain would be disrupted. On the other hand, hyperglycemia causes neuron degeneration through chronic oxidative stress (25). The current study found no significant relationship between diabetes mellitus and cognitive impairment. In contrary, other studies revealed significant relationships between diabetes mellitus and POCD (16). The severity of diabetes mellitus was not adequately analyzed in the current study; therefore, any end-organ damages due to microvascular and macrovascular changes were not elaborated in details.

One of the highlights of cardiac surgery is the application of cardiopulmonary bypass machine. Theoritically, cardiopulmonary bypass might lead to neurological problems due to inflammatory reactions and microemboli (26). In the current study, the application of cardiopulmonary bypass machine was not a strong predictor of POCD. This finding was similar with those of the past studies with cardiopulmonary bypass machine $(27,28)$. The increase of microemboli was not related to the development of POCD.

The cutoff point of duration of cardiopulmonary bypass was 120 minutes in accordance with the standard operating procedure in Cipto Mangunkusumo Hospital. Based on the demographic data, the mean duration of cardiopulmonary bypass was $118 \pm 43$ minutes. Therefore, the number of subjects with long duration of cardiopulmonary bypass was minimal. The data from the intensive care unit (ICU) showed that the mean duration of cardiopulmonary bypass in patients that died or had prolonged hospital stay was $168 \pm 55$ minutes.

Duration of cross-clamp during cardiac surgery was also believed as a predictor to POCD; during this period, the circulation is converted to synthetic circulation using the cardiopulmonary bypass machine. The application of synthetic lines and tubes causes low systemic vascular resistance (SVR). Additionally, hemodilution also occurs with low mean arteral pressure. The development of microemboli might impair cerebral blood flow (28). However, in the current study cross-clamp duration more than $90 \mathrm{~min}$ utes was not a significant risk factor for impaired cognitive function. Similar to the duration of cardiopulmonary bypass, the mean duration of cross-clamp was $89 \pm 34 \mathrm{~min}$ utes. Therefore, the difference between each category was very narrow; hence, it was not sensitive to detect any effects of cross-clamp duration on POCD.

Based on the findings of the current study, it is necessary to screen for high risk patients to develop POCD during preoperative care. This preoperative screening is especially focused on geriatric patients. Assessment of preoperative cognitive function should be conducted to understand the baseline of cognitive function. It should be thoroughly explained to the patients and their families about the possible complications including POCD.

The current study was conducted on patients that underwent cardiac surgery using general anesthesia. Therefore, further studies on different populations with different anesthesia techniques might result in different findings. The location of cognitive function test was in the ward, which was not ideal. Any distraction from the environment might affect the result of cognitive function test. The postoperative cognitive function test was conducted only once on the last day of hospitalization. However, the follow-up test should be conducted in a series of time to detect any changes in cognitive function.

There were other factors contributing to any cognitive dysfunctions following cardiac surgery. Firstly, the current study did not include the amount of postoperative consumption of sedatives and opioids postoperatively. This might contribute to altered brain function during the recovery period. Additionally, the study did not measure the duration of hypotensive period intraoperatively. Lastly, there were several comorbidities such as hypertension, hy- 
perlipidemia, carotid stenosis, and history of cardiovascular disease, which were not included in the analysis; the reason was that all subjects might have such comorbidities before surgery; therefore, these factors might not be sensitive to identify the risk of POCD. Further studies might be required to identify the effect of these factors. Additionally, the current study had limited subjects to observe any difference between on-pump and off-pump surgeries. Further randomized clinical trials should be conducted to determine whether cardiopulmonary bypass is a risk factor for POCD following cardiac surgery.

\subsection{Conclusion}

Decreased cognitive function was observed in $40.7 \%$ of the subjects that underwent cardiac surgery with cardiopulmonary bypass. Old age was a significant predictor to POCD; while education level, diabetes mellitus, duration of cardiopulmonary bypass and cross-clamp were not significant factors.

\section{Acknowledgments}

The current study was supported by the Department of Anesthesiology and Intensive Care, Faculty of Medicine, at University of Indonesia. Authors wish to thank their colleagues from the Department of Psychiatry and Division of Cardiovascular of the Department of Internal Medicine, Faculty of Medicine at University of Indonesia that provided insight and expertise that greatly assisted the study, although they may not agree with all of the interpretations/conclusions of the current paper.

\section{Footnotes}

Ethical Considerations: The study protocol was approved by the Ethical Committee of the local university.

Financial Disclosure: There was no financial conflict for the present study.

Funding/Support: The study was funded by the Department of Anesthesiology and Intensive Care, Faculty of Medicine, at University of Indonesia.

\section{References}

1. Ronawulan E. Penentuan validasi dan nilai normal uji neurokognitif[dissertation]. Jakarta: University of Indonesia; 2004.

2. Selnes OA, Gottesman RF, Grega MA, Baumgartner WA, Zeger SL, McKhann GM. Cognitive and neurologic outcomes after coronaryartery bypass surgery. NEngl J Med. 2012;366(3):250-7. doi:10.1056/NEJMra1100109. [PubMed: 22256807].
3. Boos GL, Soares LF, Oliveira Filho GR. [Postoperative cognitive dysfunction: Prevalence and associated factors]. Rev Bras Anestesiol. 2005;55(5):517-24. Portuguese. doi: 10.1590/S003470942005000500006. [PubMed: 19468642].

4. Machin D, Allsager C. Principles of cardiopulmonary bypass. Contin Educ Anaesth Crit Care Pain. 2006;6(5):176-81. doi: 10.1093/bjaceaccp/mklo43.

5. Mangano CM, Chow JL, Kanevsky M. Cardiopulmonary bypass and anesthesiologist. In: Kaplan JA, Reich DL, Savino JS, editors. Kaplan's cardiac anesthesia: The echo era. 6th ed. Philadelphia: Elsevier Saunders; 2011. p. 513-37.

6. Steidl SR. The adverse effects of the cardiopulmonary bypass machine [dissertation]. Virginia: Liberty University; 2011.

7. Tan AMY, Amoako D. Postoperative cognitive dysfunction after cardiac surgery. Contin Educ Anaesth Crit Care Pain. 2013;13(6):218-23. doi: 10.1093/bjaceaccp/mkt022.

8. Bruggemans EF. Cognitive dysfunction after cardiac surgery: Pathophysiological mechanisms and preventive strategies. Neth Heart J. 2013;21(2):70-3. doi: 10.1007/s12471-012-0347-x. [PubMed: 23184600]. [PubMed Central: PMC3547425].

9. Gao L, Taha R, Gauvin D, Othmen LB, Wang Y, Blaise G. Postoperative cognitive dysfunction after cardiac surgery. Chest. 2005;128(5):366470. doi: 10.1378/chest.128.5.3664. [PubMed: 16304328].

10. Martin JF, Melo RO, Sousa LP. Postoperative cognitive dysfunction after cardiac surgery. Rev Bras Cir Cardiovasc. 2008;23(2):245-55. [PubMed: 18820789].

11. Brown WR, Moody DM, Challa VR, Stump DA, Hammon JW. Longer duration of cardiopulmonary bypass is associated with greater numbers of cerebral microemboli. Stroke. 2000;31(3):707-13. [PubMed: 10700508].

12. Newman MF, Kirchner JL, Phillips-Bute B, Gaver V, Grocott H, Jones $\mathrm{RH}$, et al. Longitudinal assessment of neurocognitive function after coronary-artery bypass surgery. N Engl J Med. 2001;344(6):395-402. doi: 10.1056/NEJM200102083440601. [PubMed: 11172175].

13. Savage RM, Gouvier WD. Rey Auditory-Verbal Learning Test: The effects of age and gender, and norms for delayed recall and story recognition trials. Arch Clin Neuropsychol.1992;7(5):407-14. [PubMed: 14591275].

14. Griffin PT, Heffernan A. Digit span, forward and backward: Separate and unequal components of the WAIS digit span. Percept Motor Skill. 1983;56(1):335-8. doi: 10.2466/pms.1983.56.1.335.

15. Tombaugh TN. Trail Making Test A and B: Normative data stratified by age and education. Arch Clin Neuropsychol. 2004;19(2):203-14. doi: 10.1016/S0887-6177(03)00039-8. [PubMed:15010086].

16. Mu DL, Li LH, Wang DX, Li N, Shan GJ, Li J, et al. High postoperative serum cortisol level is associated with increased risk of cognitive dysfunction early after coronary artery bypass graft surgery: A prospective cohort study. Plos One. 2013;8(10). e77637. doi: 10.1371/journal.pone.0077637. [PubMed: 24143249]. [PubMed Central: PMC3797042].

17. Ziyaeifard M, Alizadehasl A, Amiri M, Rezaei H, Faiz SH, Babaee T, et al. Prevalence and predisposing factors for cognitive dysfunction following adult cardiac surgery. Res Cardiovasc Med. 2016;10. e37284. doi: 10.5812/cardiovascmed.37284.

18. Sophie S. Anaesthesia for the elderly patient. J Pak Med Assoc. 2007;57(4):196-201. [PubMed: 17489529]

19. Moller JT, Cluitmans P, Rasmussen LS, Houx P, Rasmussen H, Canet $J$, et al. Long-term postoperative cognitive dysfunction in the elderly ISPOCD1 study. ISPOCD investigators. International study of post-operative cognitive dysfunction. Lancet. 1998;351(9106):857-61. [PubMed: 9525362].

20. Jankowski CJ. Delirium and postoperative cognitive dysfunction. In: Sieber F, editor. Geriatric anesthesia.1st ed. Maryland: The Mcgraw-Hill Companies; 2007. p. 267-77. 
21. Johnson T, Monk T, Rasmussen LS, Abildstrom H, Houx P, Korttila $\mathrm{K}$, et al. Postoperative cognitive dysfunction in middle-aged patients. J Am Soc Anesthesiol. 2002;96(6):1351-7. doi: 10.1097/00000542200206000-00014. [PubMed: 12170047].

22. Leibovici D, Ritchie K, Ledesert B, Touchon J. Does education level determine the course of cognitive decline? Age Ageing. 1996;25(5):392-7. [PubMed: 8921146].

23. Kodl CT, Seaquist ER. Cognitive dysfunction and diabetes mellitus. Endocr Rev. 2008;29(4):494-511. doi: 10.1210/er.2007-0034. [PubMed: 18436709]. [PubMed Central: PMC2528851].

24. Hofmeijer J, van Putten MJ. Ischemic cerebral damage: An appraisal of synaptic failure. Stroke. 2012;43(2):607-15. doi: 10.1161/STROKEAHA.111.632943. [PubMed: 22207505].

25. Robertson RP. Chronic oxidative stress as a cenetral mechanism for glucose toxicity in pancreatic islet beta cells in diabetes. $J$ Biol Chem. 2004;279(41):42351-4. doi: 10.1074/jbc.R400019200. [PubMed: 15258147].

26. Kalkman C. Can we influence postoperative cognitive dysfunction? Acta Anaesthesiol Belg. 2007;58(4):227-9. [PubMed: 18274239].

27. Taggart DP, Browne SM, Halligan PW, Wade DT. Is cardiopulmonary bypass still the cause of cognitive dysfunction after cardiac operations? J Thorac Cardiovasc Surg. 1999;118(3):414-20. discussion 420-1. doi: 10.1016/S0022-5223(99)70177-7. [PubMed:10469952].

28. Liu YH, Wang DX, Li LH, Wu XM, Shan GJ, Su Y, et al. The effects of cardiopulmonary bypass on the number of cerebral microemboli and the incidence of cognitive dysfunction after coronary artery bypass graft surgery. Anesth Analg. 2009;109(4):1013-22. doi: 10.1213/ane.ob013e3181aed2bb. [PubMed: 19762724] 